\title{
Blood to brain to the rescue
}

\author{
Richard L. Proia and Yun-Ping Wu
}

Genetics of Development and Disease Branch, National Institute of Diabetes and Digestive and Kidney Diseases, National Institutes of Health, Bethesda, Maryland, USA.

\begin{abstract}
Neurodegeneration occurs in the majority of the more than 40 known lysosomal storage diseases. Since the nervous system in these disorders can be globally affected, effective treatment would require persistent widespread correction. Biffi et al. (see the related article beginning on page 1118) show such correction is possible in a mouse model of metachromatic leukodystrophy by the transplantation of hematopoietic cells genetically modified to overexpress the missing lysosomal enzyme. The results reveal a nervous system damage-response pathway that can be harnessed to provide therapy to the nervous system in these serious disorders.
\end{abstract}

Although individual lysosomal storage diseases are rare, as a group they represent a significant fraction of severe inherited metabolic conditions (1). The more than 40 different storage disorders cumulatively affect 1 in 5,000 live births. Most of the disorders are caused by the deficiency of a lysosomal enzyme activity that results in the progressive accumulation of the enzyme's undegraded substrates. Since lysosomal enzymes are expressed ubiquitously, many organ systems are often affected. Generally, the pathophysiology of a particular disorder is related to the degree of substrate accumulation in a cell or tissue type - determined by substrate synthesis and degradation rates - and the sensitivity of the cell or tissue type to the stored substrate. A case in point is metachromatic leukodystrophy (MLD), which is caused by the absence of arylsulfatase A. A major substrate for the enzyme is galactosyl-3-sulfate ceramide (sulfatide), a component of myelin sheaths produced by oligodendrocytes in the central nervous system and Schwann cells in the peripheral nervous system. In MLD, damage to oligodendrocytes and Schwann cells caused by storage of sulfatide results in demyelination, degeneration of the nervous system, and, in the most severe cases, death in childhood.

Neurodegeneration, a feature that MLD shares with the majority of lysosomal storage diseases, has made treatment of these

Nonstandard abbreviations used: metachromatic leukodystrophy (MLD).

Conflict of interest: The authors have declared that no conflict of interest exists.

Citation for this article:

J. Clin. Invest. 113:1108-1110 (2004).

doi:10.1172/JCI200421476. diseases exceedingly difficult. Enzyme replacement therapies have been successful in preventing or reversing the somatic manifestations of some disorders by taking advantage of the cell's efficient receptor-mediated uptake systems $(2,3)$. Lysosomal enzymes carry oligosaccharide structures that enable their recognition by plasma membrane receptors, which deliver the enzymes to lysosomes. Thus far, enzyme replacement therapy has proven unsuitable for treatment of the nervous system in storage diseases, because the blood-brain barrier precludes entry of proteins administered systemically. To circumvent this obstacle, strategies are being attempted that include direct introduction of gene transfer vectors or genetically modified cells into the nervous system (4-6). It is uncertain if such a highly invasive strategy would be suitable for the persistent and widespread correction needed for a globally affected nervous system found in many of the storage diseases. Another strategy utilizes small molecules that can pass through the blood-brain barrier to either partially inhibit substrate synthesis or stabilize the mutant enzyme $(7,8)$. While promising in concept, these approaches are in early stages.

\section{Gene therapy for the nervous system via hematopoietic stem cells}

Transplantation of hematopoietic stem cells, from either bone marrow or cord blood, has been utilized over the past several years as a potential treatment for lysosomal storage diseases. Significantly, it is the only treatment to date that has been shown to prevent or retard neurodegeneration in storage disease patients (9). A study in this issue of the JCI by Biffi et al.
(10) reinforces the concept that hematopoietic stem cell transplantation, as a therapeutic platform, has several features well-suited for the treatment of a globally affected nervous system in lysosomal storage diseases. The authors show, in a mouse model of MLD, that transplantation of hematopoietic stem cells transduced with lentiviral vectors carrying arylsulfatase A prevents neurologic disease as determined by the absence of sulfatide storage and neuropathology, and the preservation of neurologic function. Importantly, a global correction was achieved that included both the central and the peripheral nervous systems. Although hematopoietic stem cells can potentially differentiate into multiple nervous system cell types (11-14), in this study the repopulating cells were almost exclusively macrophages and microglia. Because donor cells that were engineered to produce high levels of arylsulfatase A were more effective at preventing neurodegeneration than wild-type cells, therapeutic improvement was likely achieved by enzyme-deficient cells capturing arylsulfatase A secreted by donor microglia and endoneural macrophages.

\section{Nervous system damage-response pathway}

The findings in this study (10) help clarify a damage-response mechanism relevant to the pathogenesis of lysosomal storage diseases and illustrate how it can be exploited for nervous system therapy (Figure 1). Microglia, resident macrophages of the brain, are continuously replenished by blood-borne precursors, which cross the blood-brain barrier and migrate into the parenchyma of the nervous system. They take up residence as resting microglia to surveil and defend the nervous system against damaging agents or conditions. When the nervous system is injured, as in MLD (10), a damage-response mechanism is activated that results in the enhanced recruitment of macrophages/ microglia. Most significantly, there is preferential recruitment of macrophages/microglia to sites of substrate storage and pathology $(10,14)$. Thus, if the hematopoietic stem cells have been corrected for the lysosomal 


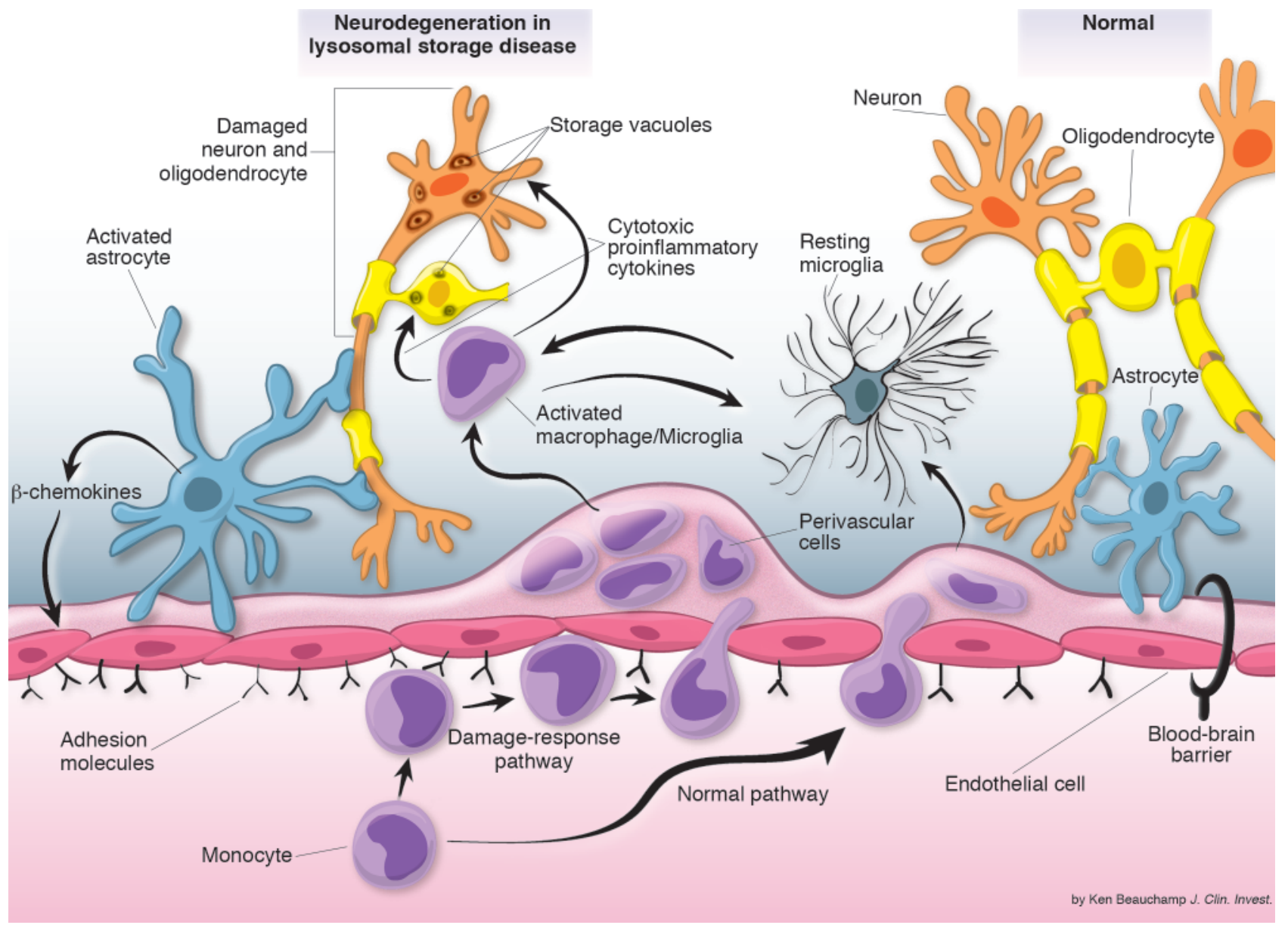

Figure 1

Damage-response pathway in lysosomal storage disease. Normally, nervous system microglia and macrophages can be derived from bloodborne precursors; they pass through the blood-brain barrier into the perivascular regions and ultimately take up residence in the parenchyma as resting ramified cells. When nervous system damage occurs, cytokines and chemokines are generated from activated macrophages/microglia and astrocytes, which can stimulate the up-regulation of adhesion molecules on nervous system endothelial cells, resulting in enhanced transendothelial migration of monocytes from the blood into perivascular regions. These enzyme-deficient cells migrate into the parenchyma to sites of damage where they may secrete potentially cytotoxic proinflammatory cytokines, leading to further damage and cell death. If the hematopoietic precursors are corrected, the macrophages and microglia gain the ability to transfer the missing lysosomal enzyme to deficient cells in the nervous system at sites of damage.

enzyme deficiency, the macrophages/microglia may be able to deliver enzyme directly to sites of damage.

The vigorous process of recruiting macrophages/microglia into the nervous system and their subsequent activation may actually hasten neurodegeneration in lysosomal storage diseases. Studies utilizing a mouse model of Sandhoff disease, another sphingolipid storage disease exhibiting profound neurodegeneration, have shown that the population of activated macrophages/ microglia expands considerably in the central nervous system prior to neuronal apoptosis (15). Intriguingly, the transplantation of these mice with bone marrow-containing hematopoietic stem cells substantially reduces macrophage/microglia activation and neuronal cell death without reducing substrate storage. Furthermore, blocking monocyte recruitment into the nervous system reduces neuronal cell death (Y.-P. Wu and R.L. Proia, unpublished data). These results indicate that the activated macrophages/microglia can trigger cell death possibly through the elaboration of cytotoxic, inflammatory mediators as has been suggested for other neurodegenerative conditions like Alzheimer disease.

The work by Biffi et al. (10) highlights the concept that therapeutic hematopoietic stem cell transplantation actually corrects a nervous system damage-response pathway defective in lysosomal storage disor- ders. Ex vivo genetic modification of hematopoietic stem cells to heighten expression of the missing enzyme in macrophages/microglia serves to restore and enhance the corrective potential of this pathway and to dampen its destructive capacity. Harnessed for therapy, the damage-response pathway appears unique in its capacity to afford widespread and persistent correction of a globally damaged nervous system as well as to target specifically affected areas. The approach taken by Biffi et al. (10), with its remarkable therapeutic effect on MLD, is particularly important because it outlines a strategy that may ultimately herald effective treatment of the nervous system in lysosomal storage diseases. 
Address correspondence to: Richard L. Proia, Building 10, Room 9N-314, National Institutes of Health, 10 Center Drive MSC 1821, Bethesda, Maryland 208921821, USA. Phone: (301) 496-4391; Fax: (301) 496-9878; E-mail: proia@nih.gov.

\footnotetext{
1. Scriver, C.R., et al., editors. 2001. In The metabolic and molecular bases of inherited disease. McGraw-Hill. New York, New York, USA. 3371-3896.

2. Brady, R.O. 2003. Enzyme replacement therapy: conception, chaos and culmination. Philos. Trans. $R$. Soc. Lond. B Biol. Sci. 358:915-919.

3. Kakkis, E.D., et al. 2001. Enzyme-replacement therapy in mucopolysaccharidosis I. N. Engl. J. Med. 344:182-188.

4. Brooks, A.I., et al. 2002. Functional correction of established central nervous system deficits in an animal model of lysosomal storage disease with feline immunodeficiency virus-based vectors. Proc.
}

Natl. Acad. Sci. U. S. A. 99:6216-6221.

5. Taylor, R.M., and Wolfe, J.H. 1997. Decreased lysosomal storage in the adult MPS VII mouse brain in the vicinity of grafts of retroviral vector-corrected fibroblasts secreting high levels of beta-glucuronidase. Nat. Med. 3:771-774.

6. Snyder, E.Y., Taylor, R.M., and Wolfe, J.H. 1995 Neural progenitor cell engraftment corrects lysosomal storage throughout the MPS VII mouse brain. Nature. 374:367-370.

7. Platt, F.M., et al. 1997. Prevention of lysosomal storage in Tay-Sachs mice treated with N-butyldeoxynojirimycin. Science. 276:428-431.

8. Fan, J.Q., Ishii, S., Asano, N., and Suzuki, Y. 1999. Accelerated transport and maturation of lysosomal alpha-galactosidase A in Fabry lymphoblasts by an enzyme inhibitor. Nat. Med. 5:112-115.

9. Krivit, W., et al. 1998. Hematopoietic stem-cell transplantation in globoid-cell leukodystrophy. N. Engl. J. Med. 338:1119-1126.

10. Biffi, A., et al. 2004. Correction of metachromatic leukodystrophy in the mouse model by trans- plantation of genetically modified hematopoietic stem cells. J. Clin. Invest. 113:1118-1129. doi:10.1172/JCI200419205

11. Eglitis, M.A., and Mezey, E. 1997. Hematopoietic cells differentiate into both microglia and macroglia in the brains of adult mice. Proc. Natl. Acad. Sci. U. S. A. 94:4080-4085.

12. Mezey, E., Chandross, K.J., Harta, G., Maki, R.A., and McKercher, S.R. 2000. Turning blood into brain: cells bearing neuronal antigens generated in vivo from bone marrow. Science. 290:1779-1782.

13. Brazelton, T.R., Rossi, F.M., Keshet, G.I., and Blau, H.M. 2000. From marrow to brain: expression of neuronal phenotypes in adult mice. Science. 290:1775-1779.

14. Priller, J., et al. 2001. Targeting gene-modified hematopoietic cells to the central nervous system: use of green fluorescent protein uncovers microglial engraftment. Nat. Med. 7:1356-1361.

15. Wada, R., Tifft, C.J., and Proia, R.L. 2000. Microglial activation precedes acute neurodegeneration in Sandhoff disease and is suppressed by bone marrow transplantation. Proc. Natl. Acad. Sci. U. S. A. 97:10954-10959.

\section{Undermining the endothelium by ablation of MAPK-MEF2 signaling}

Eric N. Olson

Department of Molecular Biology, The University of Texas Southwestern Medical Center at Dallas, Dallas, Texas, USA.

\begin{abstract}
Numerous stimuli activate Big MAPK-1 (BMK1), an MAPK that activates the myocyte enhancer factor-2 (MEF2) transcription factor. Conditional gene deletion showed BMK1 to be required for survival of endothelial cells (see the related article beginning on page 1138). An active form of MEF2C could partially bypass the requirement for BMK1 for endothelial cell survival in vitro. These findings reveal an essential role for BMK1-MEF2 signaling in an endothelial cell survival pathway and raise interesting questions about the molecular basis of this response.
\end{abstract}

A variety of extracellular stimuli transmit signals from the cell membrane to the nucleus via a cascade of MAPKs (1). MAPK pathways contain three distinct types of MAPKs that are activated sequentially (Figure 1). The most upstream kinase, MAPK kinase kinase (MEKK), is activated by $\mathrm{G}$ protein-coupled receptors and phosphorylates a subordinate MAPK kinase (MEK), which phosphorylates an MAPK. The final MAPK in the cascade phosphorylates various transcription factors, leading to activation of specific programs of gene expression.

Nonstandard abbreviations used: big MAPK-1 (BMK1); embryonic day (E); endothelial cell (EC); MAPK kinase (MEK); MAPK kinase kinase (MEKK); myocyte enhancer factor-2 (MEF2); polyinosinic-polycytidylic acid (pIpC).

Conflict of interest: The author has declared that no conflict of interest exists.

Citation for this article:

J. Clin. Invest. 113:1110-1112 (2004).

doi:10.1172/JCI200421497.
Big MAPK-1 (BMK1; also called ERK5) is the terminal MAPK that is activated by MEK5, which is activated by MEKK2/3 (Figure 1). BMK1 is unique among MAPKs because of its large size and bifunctionality. The $\mathrm{N}$-terminal region of BMK1 contains the kinase domain, while the C-terminal region functions as a transcription activation domain (2). One of the best-characterized targets of BMK1 is the myocyte enhancer factor-2 (MEF2) family of transcription factors (3-6). There are four mammalian $M E F 2$ genes, $M E F 2 A,-B,-C$, and $-D$, which are expressed in overlapping patterns in numerous cell types (7). MEF2 factors bind DNA as homo- and heterodimers and activate or repress transcription by recruiting positive or negative cofactors, many of which are cell type-specific and signal-responsive. First discovered as regulators of muscle development, MEF2 factors are now known to play diverse roles in the control of cell growth, survival, and apoptosis (7).

\section{BMK1 acts in an endothelial cell survival pathway}

Previous studies showed that KO mice lacking BMK1 die around embryonic day (E) 10 from severe abnormalities in cardiovascular development that are remarkably similar to defects seen in $\mathrm{KO}$ mice lacking MEKK3 or MEF2C, consistent with the sequential actions of these signaling molecules (8-13). However, the severity of the defects in these mutant embryos, and the associated abnormalities in yolk sac development, complicated the interpretation of the phenotypes and precluded the identification of the precise cell type responsible for embryonic lethality.

To further define the role of BMK1 in mouse development, Hayashi and coworkers generated mice harboring a conditional $B M K 1$ allele (14). In this issue of the JCI, they report that excision of $B M K 1$ after birth, using an inducible Cre recombinase transgene controlled by polyinosinic-polycytidylic (pIpC) acid, resulted in degeneration of the cardiovascular system, accompanied by multifocal hemorrhages, distended capillaries, and ruptures in the normally seamless endothelial lining of the vessels and the 